\title{
An Ascent of Big Data on Cloud: A Study
}

\author{
Gunjan Aggarwal \\ Assistant Professor \\ Sharda University \\ Greater Noida
}

\author{
Deepti Sahu \\ Assistant Professor \\ Sharda University \\ Greater Noida
}

\author{
Megha Chabbra \\ Assistant Professor \\ Sharda University \\ Greater Noida
}

\begin{abstract}
Cloud computing is an intense innovation to perform massive scale and complex computing. It wipes out the need to keep up costly processing equipment, committed space and programming. Gigantic development in the size of information or big data generated through cloud computing has been observed. The term Big Data isn't just about the extent of information that comes in pet bytes or zeta bytes; rather it is more about capability to handle huge amounts of data. Tending to big data is a challenging and time requesting task for an expansive computational framework to guarantee productive data processing and analysis. The rise of big data in cloud computing and enhancing the security of big data is reviewed in this study.
\end{abstract}

\section{Keywords}

Big Data; Data Anonymization; Cloud Computing

\section{INTRODUCTION}

Society is gradually moving towards technologies and accordingly organizations are creating and putting away immense amounts of data. Managing and extracting bits of structured information from the delivered information is a challenge and key to competitive advantage. The capacity to cross-relate private data on purchaser inclinations and data obtained from (tweets, web journals, item assessments and information from interpersonal organizations etc) opens wide variety of potential outcomes. These outcomes are useful for associations to realize the requirements of their clients anticipate their needs, requests and streamline the utilization of assets. This worldview is famously named as Big Data[1][4].Big data is a word utilized for depiction of huge amounts of data which are either organized, semi organized or unstructured. The information gathered from various events which are not taken care of by the traditional databases and programming technologies such information are manage by big data.

Big Data presents challenges to store, transport, process, mine and serve the information. Cloud computing gives basic help to address the difficulties with shared registering assets including processing, storage and networking analytical software.

Cloud computing has been reforming the IT industry by adding adaptability to the way IT is expended, empowering associations to pay just for the assets and administrations they utilize. With an end goal is to reduce IT capital and operational consumptions. Associations of all sizes are utilizing Clouds assets to run their applications. Cloud provides secure and reliable data to the clients. Mists change altogether in their particular advancements and implementation, cloud provides infrastructure, platform, and software resources as services.

\section{LITERATURE}

\subsection{Big Data}

In today's digitally connected world, each single factor will be reflection of generating data. This data that's obtaining ocean of Big Data produced from countless sources like web logs, smart phones, social network sites, satellite pictures, client transactions and biological records poses immense opportunities as well as challenges for researchers to tackle and produce useful outcome. The term Big Data is not about the magnitude of information that comes in peta bytes or zeta bytes; rather it's about the capability to handle large amounts of Knowledge [3].

Big Data requires superior information preparing tools for researchers to extract knowledge from the exceptional volume of information. A portion of the Vs used to characterize big data include variety, volume, velocity, veracity and value. One of the usually utilized models for clarifying big data is the Multi-V model.

Following are the components of Multi- $\mathrm{V}$ model.

Variety: represents various types of data

Velocity: refers to rate at which the data is created and processed

Volume: characterizes the amount of data

Veracity: refers to understandability of Big Data.

Value: relates the financial worth of Big Data.
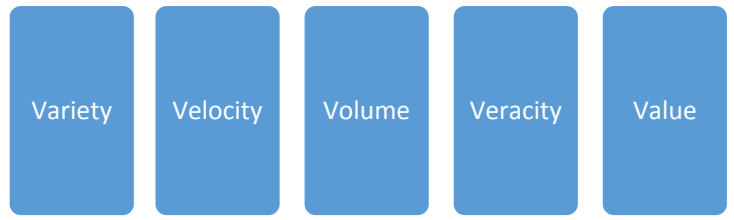

Fig 1 : Multi-V Model

The data which is very quick, has various varieties and requires sort of the well prepared structures to empower decision making, insight discovery and streamlining of process. All together to analyze the information and to distinguish patterns it is very critical for us to store, manage and share complex information on cloud. The improvement in big data applications is triggered by complex calculation which is difficult to manage [2].Big data gives a foundation to keeping up straight forwardness in assembling industry, which having the capacity to unrevealed vulnerabilities that exists in the component execution and accessibility.

Big data even enables the end clients to predict the information and enable organizations to discover new business openings. Data analytics is one of the most outstanding preferred viewpoints of the big data where the individual is permitted to customize the content or to look and feel the real time websites. Big data analytics is the process of 
examining huge amount of varied data sets i.e. big data. It is use to uncover hidden patterns, unknown correlations, market trends, customer preferences and other useful information that can help organizations to make more-informed business decisions [3].

\subsection{Cloud Computing}

Today internet is becoming an imperative tool in our day to day life, since the users are becoming abundant. In recent years cloud computing has emerged as a fundamental concept. The cloud makes use of computing resources both hardware and software which is provided in the form of a service over the internet. Mobility, huge availability and cost efficiency are the factors responsible for making cloud computing popular in today's era. On the contrary it induces more threats to the safety of the organization's statistics and information. Cloud computing gives huge computation power and storage capacity limit by means of using countless PCs together, empowering clients to send applications cost-effectively without heavy infrastructure investment. Cloud clients can decrease immense upfront investment of IT foundation and focus on their own core business. However, various potential clients are still reluctant to take advantage of cloud because of privacy and security concerns [6] [7].

Cloud computing represents both the software and the hardware delivered as services over the Internet. Cloud Computing is an emerging innovation that defines the use of computing as a utility, that has recently attracted huge consideration.

Cloud computing is composed of service models. They are following:

SaaS : Software as a Service

PaaS : Platform as a Service

IaaS : Infrastructure as a Service

\section{CHALLENGES IN BIG DATA MANAGEMENT}

Data variety: How to deal with a constantly growing volume of information? Particularly when the information is unstructured, how to rapidly extricate important substance out of it? How to aggregate and correlate streaming information from multiple sources?

Data storage: How to efficiently recognize and store important information extracted from unstructured data? How to store huge volumes of information so that it can be timely recovered? Are present document frameworks optimized for the volume and variety demanded by analytics applications? If not, what new capabilities are required? How to store information in a way that it can be effectively relocated /ported between data centers/Cloud providers?

Data integration: New protocols and interfaces for integration of information to manage data of various nature (structured, unstructured, semi- organized) and sources.

Data Processing and Resource Management: New programming models enhanced for streaming and/or multidimensional information; new backend engines that manage optimized file systems; engines able to consolidate applications from various programming models (e.g. MapReduce, workflows) on a single solution/abstraction. How to streamline asset use and vitality utilization when executing the analytics application?
To address Big Data challenges a variety of methodologies, strategies and tools are recognized to encourage the change of data into value. Computing framework, especially cloud computing, has a remarkable impact on information and knowledge extraction. Efficient handling of Big Data often requires particular innovations, such as massive parallel processing, data mining grids, distributed databases, scalable storage systems, and advanced computing architectures, foundations, platforms and structures. But the major concerns associated with this are the security and privacy issues that are significant barrier, preventing the broad adoption of public cloud. We have to discover approaches to make information secure and reliable so that we can extract useful information securely from Public Clouds.

\section{TECHNOLOGY 4.1 Data Anonymization}

Data Anonymization is the process of transforming information with the goal that it can be processed in a useful way while preventing that information from being associated to individual identities of people, objects or organizations.

Anonymization is a way that is capable of increasing the security of information to be inspected or implemented. It is characterized as the process of modifying the information that will be utilized to prevent the detection of key information. It is a procedure to convert clear text data into a non human readable and irreversible form.

$\mathrm{K}$-anonymity: It is the formal model of privacy or security that was created by L.Sweeney[13].The fundamental target of this model was to make each record unable to differentiate from a characterized number $(\mathrm{k})$ of other records if attempts are made to discover the information. A set of information is $\mathrm{k}$-anonymized if, for any record including a set properties or attributes there are minimal of (k-1) records that match those attributes. K- Anonymity offers properties to information or data attributes.

TABLE 1 :k anonymity attributes

\begin{tabular}{|l|l|l|l|}
\hline No. & AttributeType & Property & Action \\
\hline 1 & Key & $\begin{array}{l}\text { Can distinguish an } \\
\text { individual directly }\end{array}$ & $\begin{array}{l}\text { Remove or } \\
\text { obscure }\end{array}$ \\
\hline 2 & $\begin{array}{l}\text { Quasi- } \\
\text { identifier }\end{array}$ & $\begin{array}{l}\text { Can be connected with } \\
\text { external information to } \\
\text { recognize a person }\end{array}$ & $\begin{array}{l}\text { Suppress or } \\
\text { Generalize }\end{array}$ \\
\hline 3 & Sensitive & $\begin{array}{l}\text { Information that an } \\
\text { individual is sensitive } \\
\text { about revealing }\end{array}$ & $\begin{array}{l}\text { Needs to be } \\
\text { delinked thom the } \\
\text { individual }\end{array}$ \\
\hline
\end{tabular}

Example: If you attempt to identify a man from a release, but the only information you have is his date of birth and gender. There are $\mathrm{k}$ individuals that would meet the requirement. This is k-Anonymity. However $\mathrm{K}$-anonymity as a privacy model is not very successful as it is vulnerable or prone of two attacks .

- Homogeneity attack: Sensitive values in an equivalence class lack diversity.

- Background knowledge: if the attacker has background knowledge.

Data anonymization has been extensively studied and widely adopted for protection of information in sharing scenarios. Data anonymization refers to hiding identity and/or sensitive data of owners for information records. The privacy of an 
individual can be successfully saved while certain aggregate information is exposed to data users for diverse analysis and mining. A variety of anonymization algorithms with different anonymization operations have been proposed [9][10][11] [12]. In any case, the size of data sets that needs anonymization in cloud applications increases tremendously in accordance with the cloud computing and Big Data trends [5]. Data sets have turned out to be large to the point that anonymizing such data sets is becoming a considerable challenge for traditional anonymization algorithms.

\section{CONCLUSION}

Cloud computing is an absolutely necessary characteristic for present organizations to make proactive, knowledge driven decisions, as it encourages them to have future trends and behaviors predicted. Instead of insisting cloud infrastructures be thoroughly secure, we can practice potential security breaches in the cloud by anonymizing the data, making it insignificant to others while still allowing IT to process it in a useful way. In spite of the fact that data anonymization is not foolproof, it is one important tool in our continuing search of secure cloud computing. This paper provides a review of the need and utility of Big data in cloud computing. As the need for Big Data applications is growing every day, the ability to integrate them in cloud computing turns out to be more and more severe.

\section{REFERENCES}

[1] R.Devankuruchi "Analysis of Big Data Over the Years" International Journal of Scientific and Research Publications, Volume 4, Issue 1, January 20141 ISSN 2250-3153.

[2] Steve Sonaka "Big Data and the Ag sector: More than lots of numbers" International food and agribusiness review, volume 17 issue 1, 2014.

[3] A Katal, Wazid M, and Goudar R.H. "Big data: Issues, challenges, tools and Good practices.". Noida: 2013, pp. 404 - 409, 8-10 Aug. 2013.

[4] Ms.Manishasaini, Ms.PoojaTaneja, Ms.PinkiSethi "Big Data Analytics: Insights and Innovations" International
Journal of Engineering Research and Development ,Volume 6, Issue 10 April 2013 e-ISSN: 2278-067X, pISSN: 2278-800X.

[5] S. Chaudhuri, "What Next?: A Half-Dozen Data Management Research Goals for Big Data and the Cloud," Proc. 31st Symp. Principles of Database Systems (PODS '12), pp. 1-4, 2012.

[6] D. Zissis and D. Lekkas, "Addressing Cloud Computing Security Issues," Future Generation Computer Systems, vol. 28, no. 3, pp. 583- 592, 2011.

[7] H. Takabi, J.B.D. Joshi, and G. Ahn, "Security and Privacy Challenges in Cloud Computing Environments," IEEE Security and Privacy, vol. 8, no. 6, pp. 24-31, Nov. 2010.

[8] K. LeFevre, D.J. DeWitt, and R. Ramakrishnan, "Workload-Aware Anonymization Techniques for Large-Scale Data Sets," ACM Trans. Database Systems, vol. 33, no. 3, pp. 1-47, 2008.

[9] B.C.M. Fung, K. Wang, and P.S. Yu, "Anonymizing Classification Data for Privacy Preservation," IEEE Trans. Knowledge and Data Eng., vol. 19, no. 5, pp. 711725, May 2007

[10] X. Xiao and Y. Tao, "Anatomy: Simple and Effective Privacy Preservation,” Proc. 32nd Int'l Conf. Very Large Data Bases (VLDB ’06), pp. 139-150, 2006.

[11] K. LeFevre, D.J. DeWitt, and R. Ramakrishnan, "Mondrian Multidimensional K-Anonymity," Proc. 22nd Int'l Conf. Data Eng. (ICDE '06), 2006.

[12] K. LeFevre, D.J. DeWitt, and R. Ramakrishnan, "Incognito: Efficient Full-Domain K-Anonymity," Proc. ACM SIGMOD Int'l Conf. Management of Data (SIGMOD '05), pp. 49-60, 2005.

[13] L. Sweeney. K-anonymity: A model for protecting privacy. Int. J. Uncertain. Fuzz., 10(5):557-570, 2002 